Article

\title{
Analytical and Experimental Evaluation of a Novel Mechanism to Improve the Control Plane in Next-Generation Mobile Networks
}

\author{
Jesús Calle-Cancho ${ }^{1, *} \mathbb{C}^{\mathbb{D}}$, David Cortés-Polo ${ }^{1}{ }^{\mathbb{D}}$, José-Luis González-Sánchez ${ }^{1} \mathbb{1}$, \\ Luis Ignacio Jiménez ${ }^{1}\left[\right.$ and Javier Carmona-Murillo ${ }^{2} \mathbb{D}$ \\ 1 Research, Technological Innovation and Supercomputing Center of Extremadura (CénitS), \\ 10071 Cáceres, Spain; david.cortes@cenits.es (D.C.-P.); joseluis.gonzalez@cenits.es (J.-L.G.-S.); \\ luisignacio.jimenez@cenits.es (L.I.J.) \\ 2 Department of Computing and Telematics Engineering, University of Extremadura, 10003 Cáceres, Spain; \\ jcarmur@unex.es \\ * Correspondence: jesus.calle@cenits.es
}

Received: 6 February 2020; Accepted: 27 February 2020; Published: 29 February 2020

check for updates

\begin{abstract}
With the continuous development of mobile communications and the Internet of Things technology, the enhanced network performance can be seen as one of the major challenges in the fourth industrial revolution context, where new services and applications with strict performance requirements have emerged, such as driverless vehicles, smart cities, factories, and manufacturing, among others. These new services and applications also drive growth of the data traffic, which is increasing exponentially. Thus, in mobile network environments, industry and academia are proposing new mechanisms to overcome the traffic bottlenecks and reduce the signaling overhead that affects current networks. Centralized Mobility Management solutions are prone to several problems such as the aforementioned signaling overhead or scalability issues. To overcome these limitations, Distributed Mobility Management approaches are being considered. In this paper, an analytical cost model and experimental evaluation will be developed for evaluating the performance of the Distributed Mobility Management implementations. Furthermore, a new approach will be proposed to improve network performance.
\end{abstract}

Keywords: networked control; control plane; distributed mobility management; experimental evaluation

\section{Introduction}

Over the last years, the Internet has transformed the telecommunications industry and will continue to do so because the number of devices are increasing. Furthermore, mobile data traffic has experimented an exponential growth due to the proliferation of these smart mobile devices and the emergence of Internet of Things (IoT). All these changes are impacting on communications networks environments. According to a recent forecast, there will be 28.5 billion networked devices by $2022,37 \%$ greater compared with 2017. In addition, global mobile data traffic will increase sevenfold between 2017 and 2022, reaching 77.5 exabytes per month by 2022 [1]. The signaling overhead is expected to increase almost $50 \%$ faster than the growth in data traffic [2].

With the emergence of interconnected devices and services, the IoT has been touted to become the next major extension to the current fixed and mobile networking infrastructures. In this heterogeneous environment, the challenge is the provisioning of adequate mobility management to control and exploit realistic mobility of both IoT devices and real-world entities. 
Thus, a large number of these devices are mobile, and therefore they require a seamless mobility management mechanism during its connection to the network. Mobility support is an active research field in communications and several protocols have been proposed. In next-generation mobile network environments, user connectivity is maintained by the mobility management protocols, which provide seamless mobility support at the network level by maintaining ongoing communications while the users roam among different access networks. These network layer solutions do not rely on or make any assumptions about the underlying wireless access technologies.

To provide the continuous communication for mobile devices, the Internet Engineering Task Force (IETF) standardized different mobility management protocols such as Mobile IPv6 (MIPv6) [3] or Proxy Mobile IPv6 (PMIPv6) [4], to be adopted in the mobile network architectures. MIPv6 is probably the most widely known IP mobility support protocol, and it provides the mobility functionalities for an $\mathrm{MN}$ away from its home network by updating its TCP/IP stack. This means that the MN is responsible for all mobility-related signaling. Unlike the MIPv6 host-based approach, in a network-based mobility management approach such as PMIPv6, the serving network handles the mobility management on behalf of the $\mathrm{MN}$; thus, the $\mathrm{MN}$ is not required to participate in any mobility-related signaling. Therefore, these solutions are based on the existence of a central entity responsible for managing the movement of the Mobile Node (MN) when it is outside of its home network. This central agent is responsible to maintain the location of the $\mathrm{MN}$ and redirect the traffic to them.

Nevertheless, these Centralized Mobility Management (CMM) paradigms are not efficient when handling a large volume of mobile data traffic. These CMM architectures have certain problems and limitations, such as non-optimal routes, centrally deployed mobility anchors (single point of failure), and reliability and scalability issues [5,6]. To address these problems, new Distributed Mobility Management (DMM) protocols are being proposed [6,7] in which the mobility anchors are located closer to the user with the aim of achieving a flatter network. Thus, the main concept behind DMM solutions is the placement of the mobility agent as close as possible to the MN. Moreover, DMM reduces the traffic bottlenecks that affect current mobile networks due to the increase in data traffic with the emergence of IoT technology, new services, and applications.

The most representative distributed mobility management protocol is Network-Based DMM (NB-DMM) [7], which shares with PMIPv6 the fact that it is network-based. It exempts the MN from participating in any mobility signaling, so no network software upgrade is required at the MN for mobility support, because distributed mobility anchors perform mobility signaling on behalf of the $\mathrm{MN}$. The mobility management functionalities are moved to the DMM gateway level to anchor the traffic closer (topologically) to the $\mathrm{MN}$, distributing the control and data plane mobility functions among theses entities. The operation of NB-DMM is depicted in Figure 1.

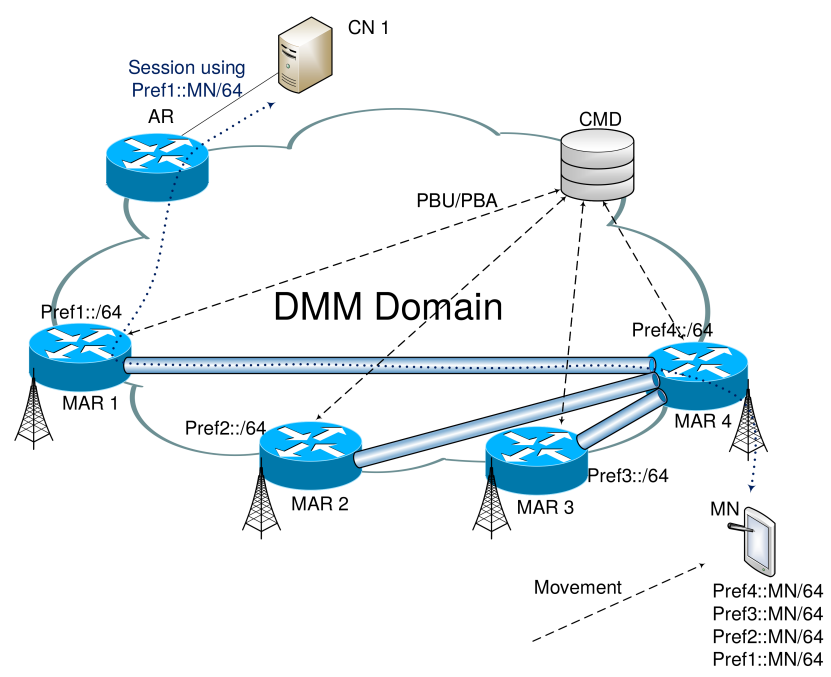

Figure 1. Network-based Distributed Mobility Management (DMM) architecture. 
This work is focused on the analysis and optimization of these network-based DMM solutions. These NB-DMM solutions have a problem regarding the signaling load of the access network. In this article, we present a proposal to improve the control plane in DMM networks, called Tunneling Extension to Distributed Mobility Management (TE-DMM). In addition, an analytical and experimental comparison is conducted to show the benefits of our proposal. TE-DMM is evaluated in terms of signaling and processing costs, handover latency, and packet loss.

This article is structured as follows. Section 2 presents the TE-DMM proposal related to the efficient management of the control plane. An analytical comparison between NB-DMM and the proposal presented in this article is provided in Section 3. Section 4 describes the implementation of TE-DMM and the experimental evaluation is performed on a real testbed. Finally, some conclusions are presented in Section 5.

\section{TE-DMM Proposal}

Network-based DMM approaches mainly introduce two functional entities called Mobility Access Router (MAR) and Context Mobility Database (CMD) [7,8], as shown Figure 1. Each MAR acts as a mobility agent located on the edge of the access network providing both mobility anchoring and location functionalities, and it is referred to as a mobility capable access router. These mobility agents are responsible for maintaining the sessions active when an $\mathrm{MN}$ moves between different network domains, providing the detection and negotiation of the handover at the IP level. When a handover occurs, the data traffic of each session is tunneled between the previous MAR and the serving MAR for this session. To achieve a network-based solution without the participation of the MN in the mobility signaling, the architecture is totally distributed and relies on a CMD. This database stores ongoing sessions for the MNs; it stores the home network prefix currently allocated to the MN and their respective anchoring points.

The main goal of our proposal is to address the problems that appear in distributed mobility management approaches from the control plane by reducing signaling load and, consequently, handover latency and packet loss.

In order to achieve that goal, TE-DMM introduces a new entity called Tunneling-Mobility Access Router (T-MAR), which acts as a MAR node, but it modifies the tunneling management required by an MN that performs its movement by the TE-DMM domain. This new procedure provides great benefits in the control plane, reducing the signaling traffic. Figure 2 shows the basic operation of TE-DMM.

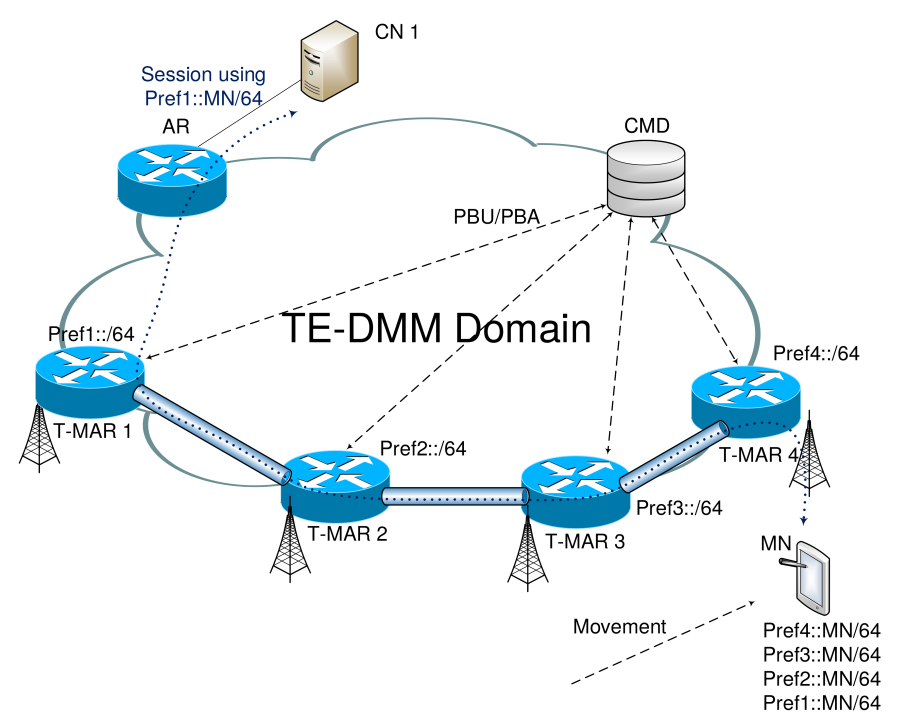

Figure 2. Basic operation of Proposed Network Architecture: Tunneling Extension to Distributed Mobility Management (TE-DMM). 
In the NB-DMM solution, once the MN performs the movement, a router solicitation message (RS) is sent from MN to the serving MAR (1), which is responsible for notifying the CMD by sending a Proxy Binding Update (PBU) message (2). When this message is received, the CMD replies with a Proxy Binding Acknowledgement (PBA) message (3). The serving MAR retrieves the IP addresses of the anchoring MAR for the MN's sessions from the database. Then, the serving MAR proceeds to update the location by sending a PBU to each anchoring MAR (4). Each anchoring MAR replies by a PBA. In addition, the tunnels between MAR are updated (5). Figure 3 shows the messages flow diagram of NB-DMM solution.

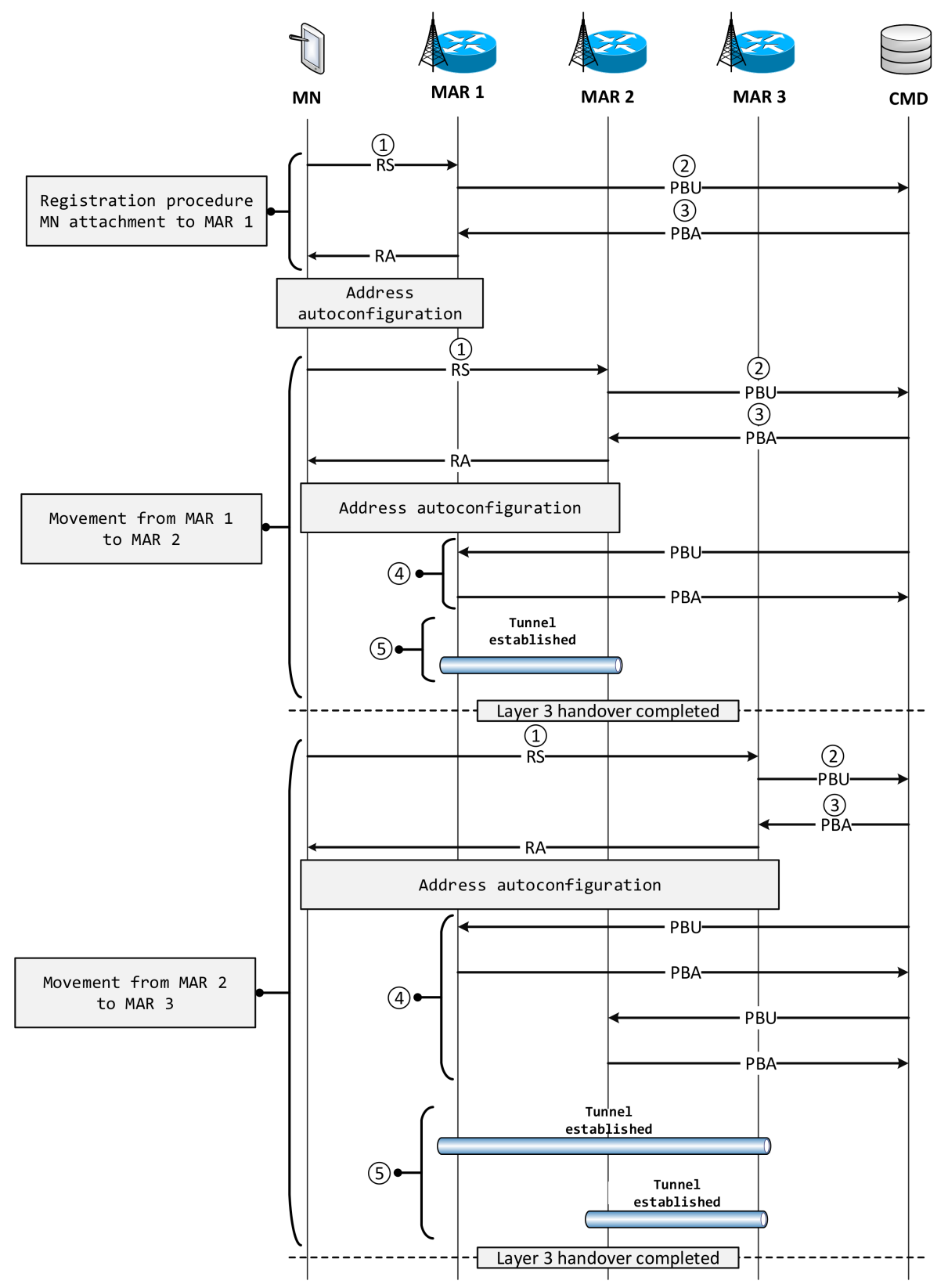

Figure 3. Messages flow in NB-DMM.

Nevertheless, in TE-DMM, tunnel management is optimized by reducing the number of tunnels that need to be established during the movement of an MN. The main concept is to extend a single tunnel from the original T-MAR to the T-MAR in which the MN is located. These tunnels are responsible for properly handling data plane traffic. The messages flow diagram of TE-DMM is shown in Figure 4. 


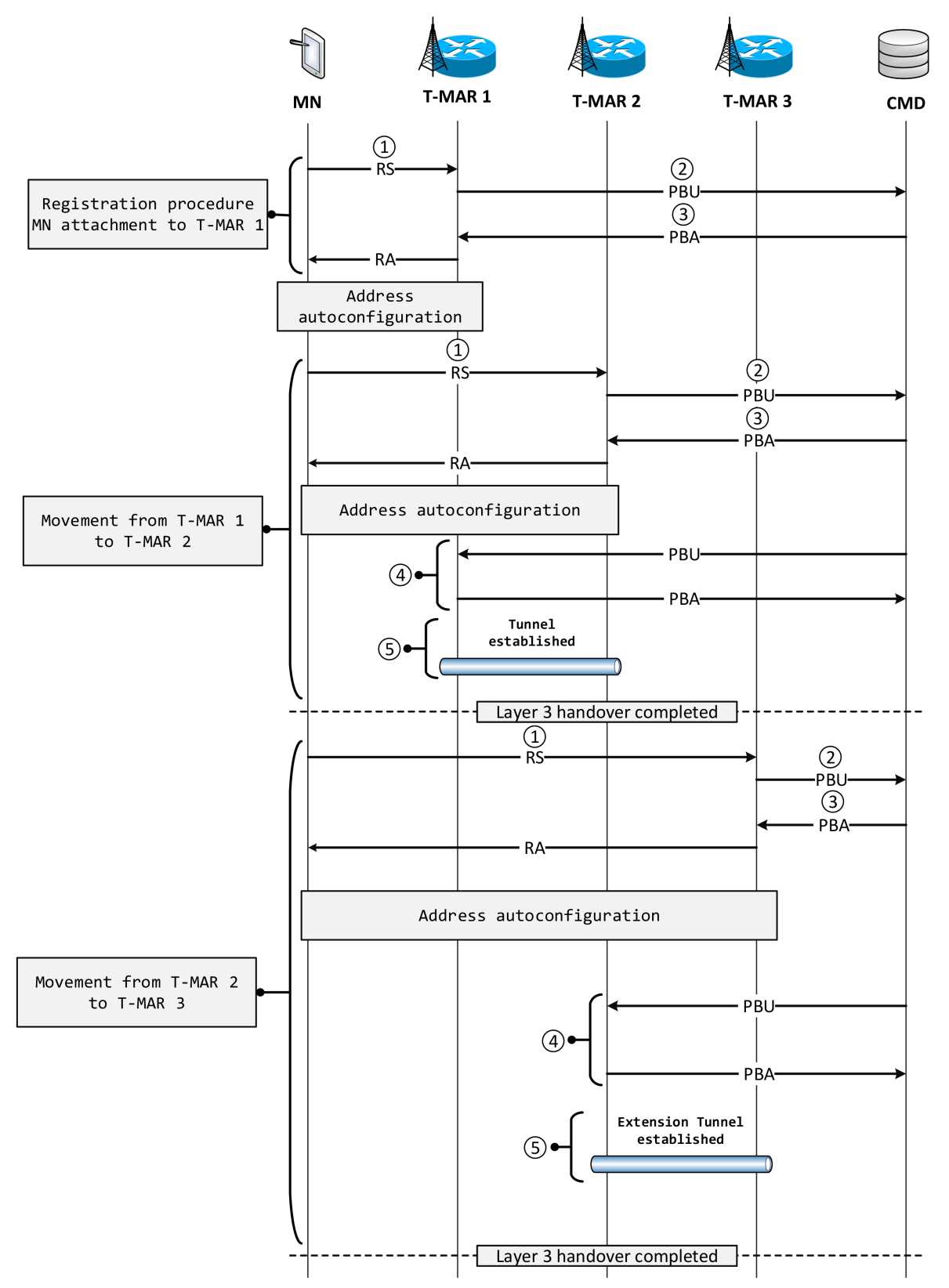

Figure 4. Messages flow in TE-DMM.

The basic operation of TE-DMM from the control plane could be described as follows. When the MN performs a handover, it sends an RS to the access T-MAR (1), which is providing service, notifying the CMD of the association through a PBU (2). When this message is received at the CMD, the information of that $\mathrm{MN}$ is updated. Moreover, CMD sends a proxy binding acknowledgment message to the corresponding T-MAR (3), as well as a PBU to the previous T-MAR (4) to perform the tunnel extension (5) correctly. As can be seen, the main difference is that must not notify all previous T-MAR, but only the last one, reducing the signaling and time loads. Thus, it is only necessary to establish a tunnel with the previous T-MAR.

\section{Analysis and Performance Evaluation}

In this section, an analytical model is developed to evaluate the performance of distributed mobility management (NB-DMM) solution and our proposal (TE-DMM) comparatively, in terms of signaling costs, processing costs, and packet loss. 
We consider a scenario where a MN might be actively engaged simultaneously with several correspondent nodes on the Internet, i.e., having several active sessions. It is assumed that the number of incoming sessions per mobile user follows a homogeneous Poisson process with mean rate $\lambda_{S}$ (i.e., the inter-arrival time between sessions is exponentially distributed with this rate). Thus, for traffic modeling, a queuing system $\mathrm{M} / \mathrm{M} / \infty[6,8,9]$ is used, in which, as previously mentioned, the incoming sessions following a Poisson process with a $\lambda_{S}$ rate. We assume also that the duration of a session is exponentially distributed with parameter $\mu_{S}[10,11]$. Therefore, by modeling the scenario as a system under the probability distribution of a typical $\mathrm{M} / \mathrm{M} / \infty$ queue, the average number of active sessions $\left(\rho_{S}\right)$ at a time is expressed as follows.

$$
\rho_{S}=\frac{\lambda_{S}}{\mu_{S}}
$$

With respect to the mobility model, we have used a model commonly used in the literature, called Fluid-Flow Model [12-14]. This model is widely used to characterize the average residence time of an $\mathrm{MN}$ in a specific subnet. Thus, the movement of a MN is represented, considering the handover rate as the average number of crossings of the region for a given area. The model assumes that the density of users is uniformly throughout this area, and that the direction with respect to the border is uniformly distributed over the range $[0,2 \pi)$. Let $v$ be average speed of the $\mathrm{MN} ; L_{S}$ and $A_{S}$ are the perimeter and area of each region, respectively. Therefore, the cell crossing rate $\mu_{S R}$ can be expressed as

$$
\mu_{S R}=\frac{v \cdot L_{S}}{\pi \cdot A_{S}}
$$

In Figure 5, the cell distribution in the coverage area is shown. Each cell is surrounded by rings of cells. Cell 0 is considered as the center cell; cells labeled 1 form the first ring around cell 0 , and so on. Each ring is labeled according to its distance from the center [12]. Thus, $a_{i}$ refers to the $a$ th ring away from the center cell, and it is shown in Figure 5 the number of cells in $a$ th ring is $6 k$. The total number of cells $N(k)$ in a coverage area is defined in Equation (3).

$$
N(k)=\sum_{i=1}^{k} 6 \cdot k+1=3 \cdot(k+1) \cdot k+1
$$

Consequently, given that the cell side $l$, we can define $L_{S}$ and $A_{S}$, as is shown below in Equation (4). Moreover, we can also defined the cell crossing rate as shown in Equation (5).

$$
\begin{aligned}
& L_{S}=6 \cdot l \quad A_{S}=\frac{3 \cdot \sqrt{3} \cdot l^{2}}{2} \\
& \mu_{S R}=\frac{v \cdot L_{S}}{\pi \cdot A_{S}}=\frac{v}{l} \cdot\left(\frac{4 \cdot \sqrt{3}}{3 \cdot \pi}\right)
\end{aligned}
$$

Likewise, the average cell residence time $T_{S R}$ for a mobile node can be calculated as shown in Equation (6).

$$
T_{S R}=\frac{1}{\mu_{S R}}
$$




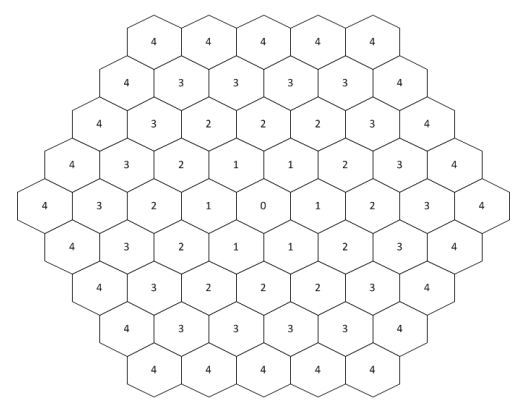

Figure 5. Distribution and number of cells in a coverage area.

\subsection{Signaling Cost}

The process of ensuring the mobility session is up to date while an MN moves among networks is the one of the main functionalities for any mobility management protocol. This task requires control messages that need to be sent among the mobility agents in the network. Therefore, the total signaling cost during a session is denoted by $C_{S}$. This cost depends on the size of the signaling messages and the number of hops in each $\mathrm{L} 3$ handover process during the time interval that the $\mathrm{MN}$ communication remains active.

In NB-DMM, $C_{S}(D M M)$ can be calculated as the cost to update the mobility entry of the MN in the CMD. Moreover, the serving MAR retrieves information of all previous MAR and it establishes bidirectional tunnels with them. Therefore, the signaling cost during MN movement for NB-DMM solution is summarized in the following expression,

$$
C_{S}(D M M)=\mu_{S R} \cdot\left(C_{C M D}+\sum_{i=1}^{n-1}\left(C_{M A R}\right)_{i}\right)
$$

where $n$ defines to the number of previous MAR that have to establish a tunnel with the serving MAR.

Moreover, the update cost of the MAR entity $\left(C_{M A R}\right)$ is defined in Equation (8).

$$
C_{M A R}=2 \cdot s_{C} \cdot h_{C M D-M A R}
$$

where $s_{\mathcal{C}}$ is the average size of the control messages and $h_{C M D-M A R}$ is the distance in number of hops from CMD to MAR.

However, in TE-DMM $\left(C_{S}(T E-D M M)\right)$, the CMD is updated and the registration update is only required with the previous T-MAR. Thus, it is only necessary to establish a tunnel with the previous T-MAR. Therefore, the signaling cost for TE-DMM solution is defined as follows.

$$
C_{S}(T E-D M M)=\mu_{S R} \cdot\left(C_{C M D}+C_{T M A R}\right)
$$

The update cost of the T-MAR entity $\left(C_{T M A R}\right)$ is described in Equation (10).

$$
C_{T M A R}=2 \cdot s_{C} \cdot h_{C M D-T M A R}
$$

where $s_{c}$ is the average size of the control messages and $h_{C M D-T M A R}$ is the distance in number of hops from CMD to T-MAR.

For both NB-DMM and TE-DMM solutions, the cost to update the mobility entry on the CMD is expressed as

$$
C_{C M D}=2 \cdot s_{C} \cdot h_{M A R-C M D}=2 \cdot s_{C} \cdot h_{T M A R-C M D}
$$

where $s_{c}$ is the average size of the control messages and $h_{x-y}$ is the distance in number of hops from the node $x$ to node $y$ in the network domain. 


\subsection{Processing Cost}

The processing $\operatorname{cost} C_{P}$ represents the number of signaling messages processed by network entity per unit time [8].

This metric is relevant because its high values reflect a higher probability of having scalability problems, which are a major concern in current mobility protocols. Therefore, we have considered evaluating the cost of processing associated with the CMD. The interest in this entity is due to the fact that it is a common point to store all mobility sessions entries for the mobile nodes of a given network domain.

In short, for both proposals, $C_{P}$ is considered as the number of signaling messages processed per unit time in the CMD for a given MN, as shown in Equations (12) and (13).

$$
\begin{gathered}
C_{P}(D M M)=2 \cdot n \cdot \mu_{S R} \\
C_{P}(T E-D M M)=4 \cdot \mu_{S R}
\end{gathered}
$$

\subsection{Packet Loss}

The handover latency $L H$ is a critical metric that has a huge impact on the performance of the system. It can be defined as the interval time in which an MN does not have connectivity as a result of a handover. This handover process is produced when an MN changes its point of attachment to the network and a disruption time exists. $L H$ consists of 22 handover latency $\left(T_{L 2}\right)$, the time needed to detect the movement $\left(T_{D M}\right)$, and the time to update the active sessions $T_{A C}$, as shown in Equation (14).

$$
L H=T_{L 2}+T_{D M}+T_{A C}
$$

Related with the handover process, other relevant metric is the packet loss during handover latency. In fact, the number of packet loss during a handover is directly proportional to the handover latency and the packet arrival rate $\left(\mu_{S} \cdot P_{S}\right)$. Therefore, the packet loss $(P L)$ can be calculated as shown in Equation (15).

$$
P L=\rho_{S} \cdot \mu_{S} \cdot P_{S} \cdot L H
$$

where $\rho_{S}$ is the average number of active sessions, $\mu_{S}$ is the duration of a session, and $P_{S}$ is the average number of packets in a session.

\subsection{Numerical Results}

In this section, the numerical results obtained analytically are presented and discussed. Both proposals NB-DMM and TE-DMM are evaluated in terms of signaling cost, processing cost, and packet loss. Additionally, the network performance is also evaluated in experimental testbed through handover latency measurements. Thus, we study the impact of several parameters on the mobility costs.

The following default parameters are the basic configuration of the topology, mobility, and traffic models used in the analysis $[6,8,11,15,16]$.

- Side of the hexagonal cells: $l=500 \mathrm{~m}$.

- Velocity of the MN: $v=20 \mathrm{~m} / \mathrm{s}$.

- Size of control messages: $s_{c}=80$ bytes.

- Number of hops between network entities: $h_{M A R-C M D}=h_{C M D-M A R}=h_{T M A R-C M D}=$ $h_{C M D-T M A R}=12$ hops.

- Number of MAR/T-MAR into the network topology: $n=3$.

- Average number of packets in a session: $P_{S}=1000$.

- Duration of a session: $1 / \mu_{S}=20 \mathrm{~s}$.

- Number of incoming sessions per $\mathrm{MN}: \lambda_{S}=40 / 3600 \mathrm{~s}^{-1}$. 
Figure 6 shows the comparison of signaling cost as a function of the velocity of the MN, which varies from 5 to $25 \mathrm{~m} / \mathrm{s}$. Thus, the impact of the MN velocity is evaluated under the same network conditions. Moreover, we have considered several numbers of mobility agents that vary between 3 and 5. This effect has been studied for the NB-DMM proposal as it is the only one that depends on the MAR number. In TE-DMM, the registration update is only needed with the previous MAR.

Therefore, the signaling cost is directly proportional to the $v$ value for both NB-DMM and TE-DMM solutions, being always lower in TE-DMM due to the efficient management of the control plane.

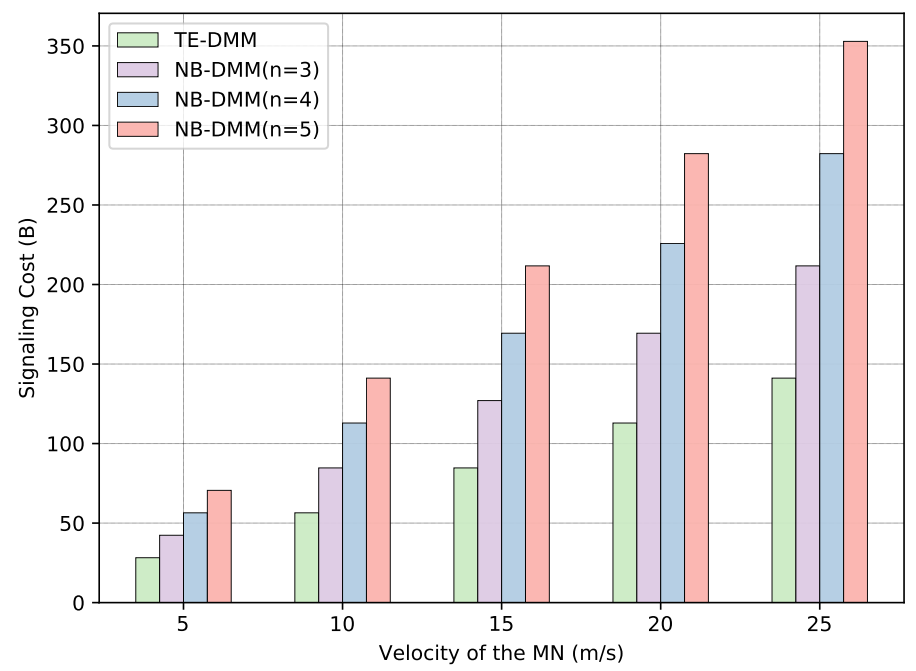

Figure 6. Signaling cost depending on velocity of the MN.

Regarding the signaling cost, the effect of the side of the hexagonal coverage cell $(l)$ has also been evaluated, varying the side of the hexagonal cells between 400 and 800 meters, as shown in Figure 7 . We consider the default values mentioned above except that of $l$. As can be observed in Figure 7, when the side of the hexagonal coverage cell increases, the signaling cost decreases because the handover rate decreases proportionally. This results in a decrease in updates of $\mathrm{MN}$ active sessions on mobility agents. Moreover, we observe an enhanced signaling cost of our proposal (TE-DMM) when the value of the side of the hexagonal cell is increased. Thus, the signaling cost in TE-DMM is lower than the signaling cost introduced by NB-DMM.

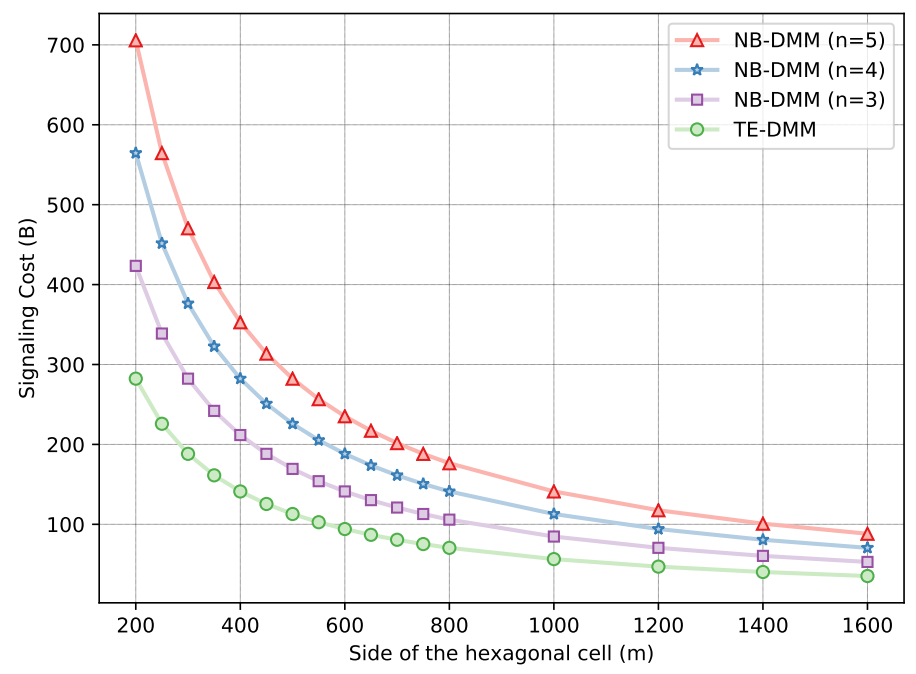

Figure 7. Signaling cost depending on side of the hexagonal cell. 
Furthermore, the processing cost has been evaluated in relation to the number of active MAR for an MN. In Figure 8, the variation of this processing cost is shown as a function of the number of MAR. As can be observed, the processing cost in NB-DMM is higher than in TE-DMM proposal. Figure 8 shows that in NB-DMM, the processing cost is higher when the number of MAR increases. However, with TE-DMM, the value remains constant because it is not necessary to update previous T-MARs, only the last one, reducing the time loads. Thus, TE-DMM achieves significant gains in terms of processing cost and signaling cost, providing substantial improvements in the control plane.

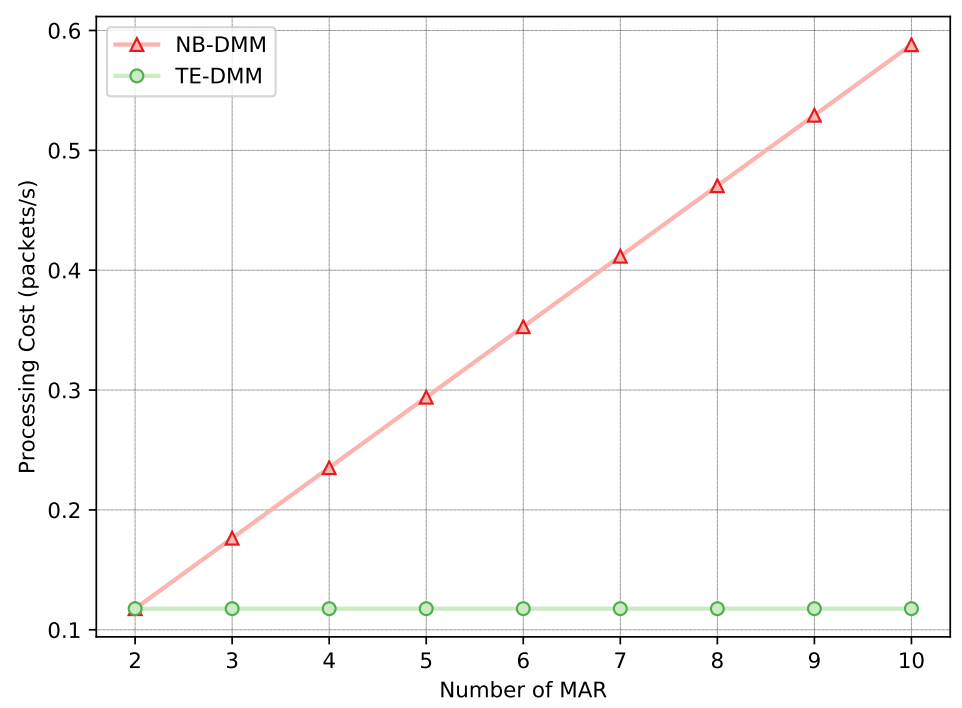

Figure 8. Processing cost depending on number of mobility anchors.

Figure 9 shows the comparison of packet loss as a function of the average number of packets in a session $\left(\rho_{S}\right)$. As can be observed, the results confirm that the packet loss increases with the number of packets in a session. These results show a linear trend in both solutions, but TE-DMM shows better performance than DMM.

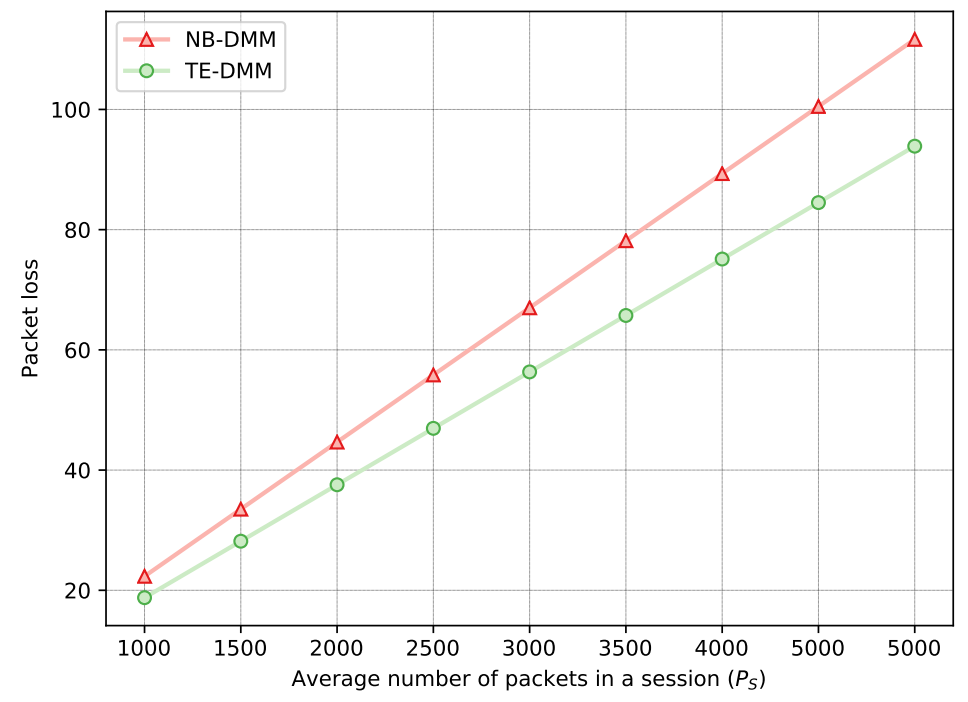

Figure 9. Packet loss depending on number of of packets. 


\section{Experimental Results}

This section presents an experimental evaluation using real implementations of NB-DMM and our solution TE-DMM. NB-DMM has been analyzed using a implementation for Linux systems called MAD-PMIPv6 (Mobility Anchors Distribution for Proxy Mobile IPv6) [17], which is developed in C language over the Linux kernel.

On the other hand, our proposal TE-DMM is based on MAD-PMIPv6. The mechanism of tunneling extension has been implemented in C language and it also runs on the Linux kernel.

The performance evaluation has been conducted on the testbed depicted in Figure 10. Thus, both solutions have been evaluated on the same network topology, providing a more reliable comparison.

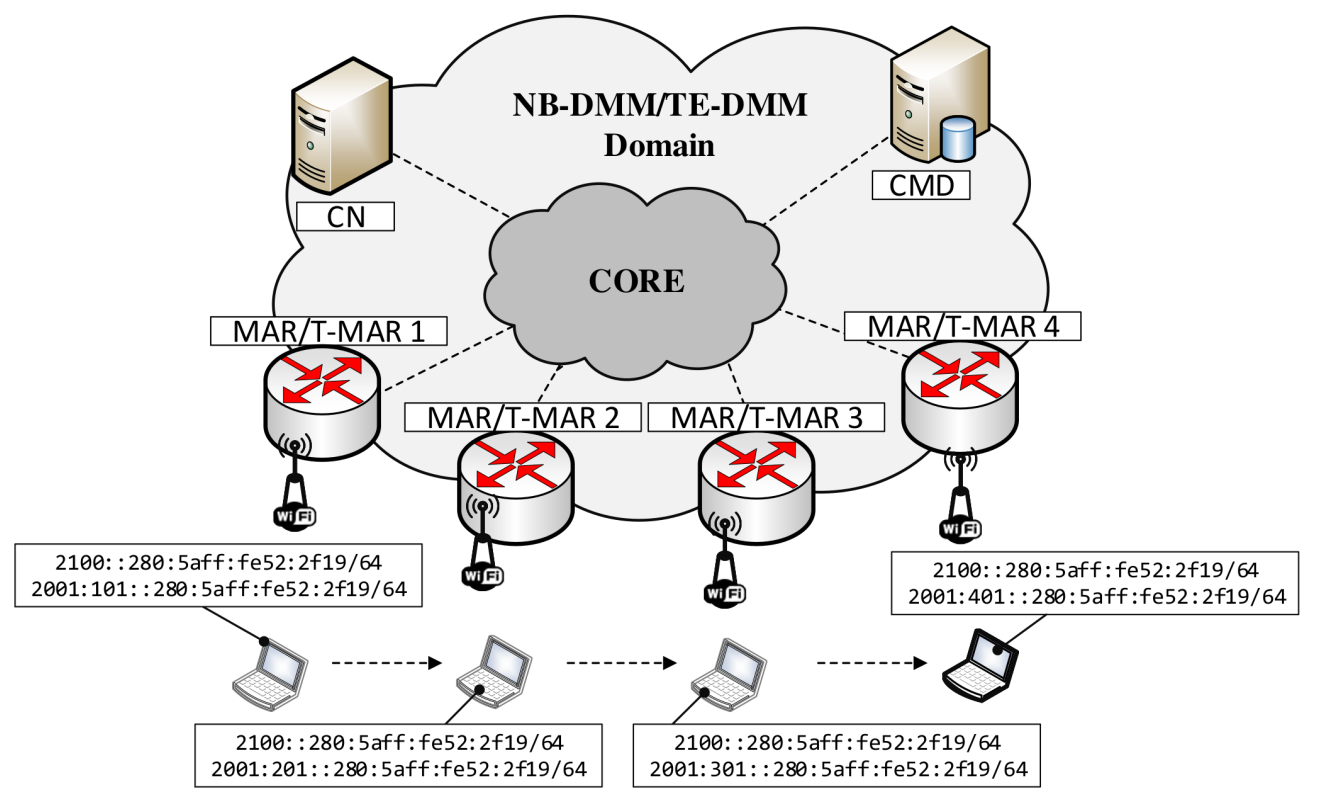

Figure 10. Testbed used in experimental evaluation.

The access network consists of four mobility anchors (MAR/T-MAR), which provide access to the mobile nodes through wireless interfaces by using IEEE $802.11 \mathrm{~g}$ technology. Moreover, the backbone of the network is emulated using CORE [18]. Therefore, this backbone interconnects all mobility agents, the $\mathrm{CMD}$, the Correspondent Node $(\mathrm{CN})$, and the access points, which provide connectivity to the mobile nodes. Experimental evaluation has been conducted using the supercomputing facilities of Research, Technological Innovation and Supercomputing Center of Extremadura (CénitS) (Technical features of LUSITANIA II: http:/ / www.cenits.es/en/cenits/lusitania-II/lusitania-ii-specifications). Each entity runs on a computer with 80 GB RAM and CPU Intel Haswell x86 (20 cores) at $2.6 \mathrm{GHz}$.

NB-DMM and TE-DMM require the TCP/IPv6 stack of all devices to be updated. Mobility anchors (MAR/T-MAR) and CMD run with a compiled Linux kernel with mobility features. However, the CN and the MN use a Linux OS that does not require any changes to the kernel or additional software.

The experimental evaluation focuses on the handover latency and the packet loss. The handover latency is defined as the time interval in which an MN does not have IP connectivity as a result of a handover process and a disruption time exits. In fact, the number of packets lost during a handover is directly proportional to the handover latency.

In the experimental evaluation, we use Wireshark at the $\mathrm{MN}$ to extract the events produced when repeating the following sequence; the MN attaches to MAR/T-MAR 1 and $\mathrm{CN}$ starts an UDP stream to the MN. Then, the MN visits MAR/T-MAR 2, MAR/T-MAR 3, and MAR/T-MAR 4 maintaining the initial active flow associated with the first anchor. Then, the MN comes back to MAR/T-MAR 1 and 
repeats the process which is repeated obtaining more than 300 handovers for both solutions. Figure 11 depicts the empirical cumulative distribution function $(\mathrm{CDF})$ for the values of the UDP stream recovery time in the testbed for NB-DMM and TE-DMM.

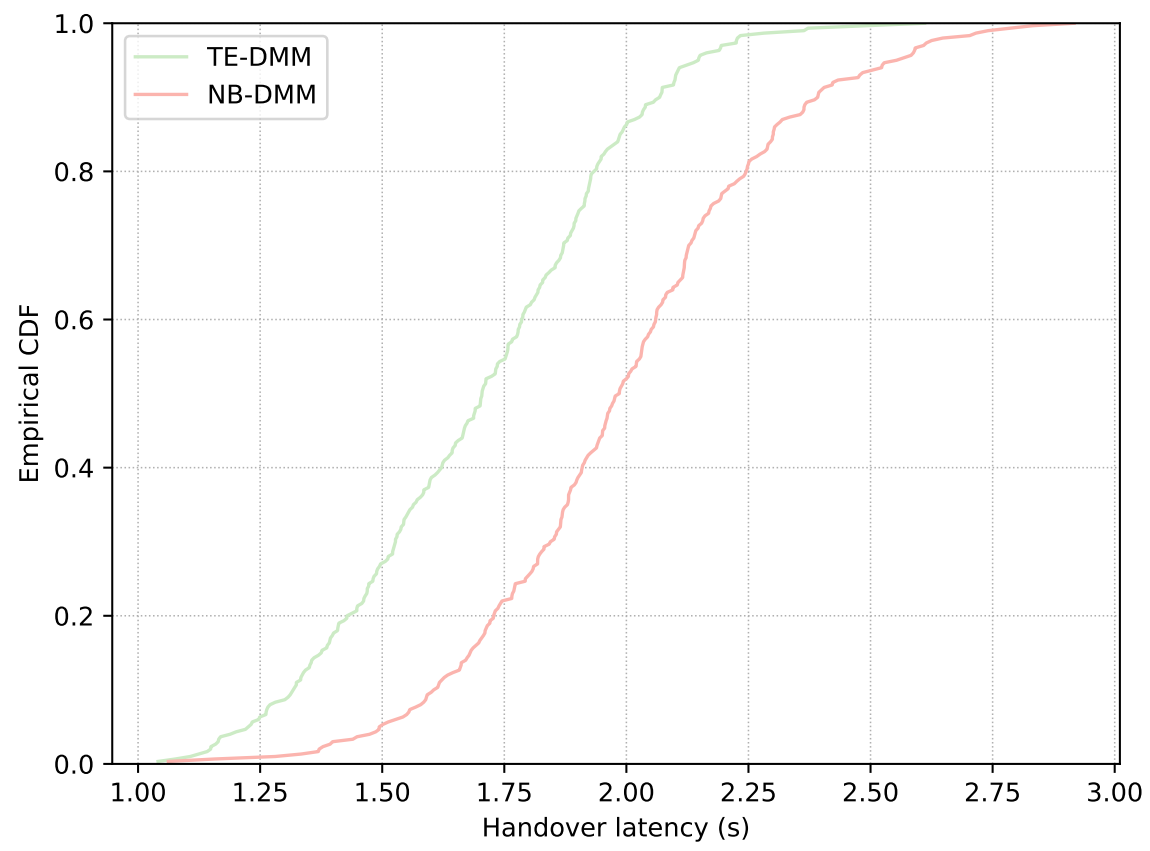

Figure 11. Empirical cumulative distribution function $(\mathrm{CDF})$ of the handover measurements.

Figure 12 shows the packet loss calculated by Equation (15) for both proposals, using the mean values of the handover latency.

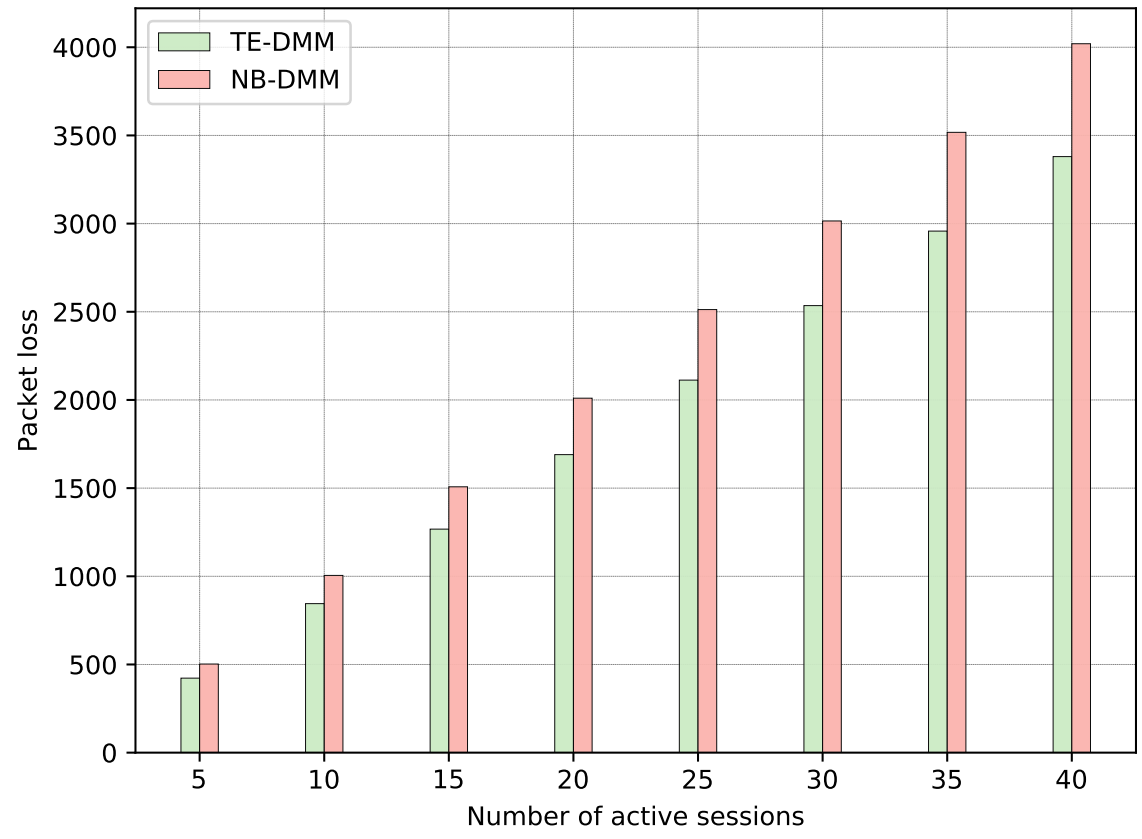

Figure 12. Packet loss evaluation: DMM and TE-DMM. 
Thus, as can be observed, Figure 12 shows the comparison of packet loss as a function of the average number of active session $\left(\rho_{S}\right)$. The packet loss in TE-DMM is lower than in NB-DMM because it is not necessary to update all the previous T-MAR, reducing the handover latency and the packet loss.

\section{Conclusions}

This article presents a novel approach called TE-DMM to reduce the signaling load, the handover latency, and the packet loss. The approach is a simple and cost-effective packet-based access network solution, which introduces a new entity that improves the tunneling management required by the MN to maintain the IP connectivity. This procedure improve the control plane and reduces the signaling traffic which must be transported by the network.

An analytical and experimental performance evaluation have been also conducted in this paper. The analytical model have demonstrated the benefits introduced by TE-DMM in terms of signaling cost which reduces in around a 50\% compared with NB-DMM. Moreover, the analytical also reveals that TE-DMM reduces the complexity of the control plane improving the tunneling managements process during the movement of the users.

Besides, an experimental evaluation using real implementations of NB-DMM and our mechanisms (TE-DMM) has been conducted in a mobility testbed. The obtained results show that TE-DMM can alleviate the handover latency providing significant benefits on the packet loss.

Author Contributions: Conceptualization, J.C.-C.; Methodology, J.C.-C., D.C.-P., and J.C.-M.; Software, J.C.-C.; Funding acquisition, J.-L.G.-S.; Validation, J.C.-C.; Formal analysis, J.C.-C., D.C.-P., and J.C.-M.; Investigation, J.C.-C., D.C.-P. and J.C.-M.; Resources, J.C.-C. and J.-L.G.-S.; Data Curation, J.C.-C.; Writing-original draft preparation, J.C.-C.; Writing-review and editing, J.C.-C., D.C.-P., J.-L.G.-S., L.I.J., and J.C.-M. ; Supervision, D.C.-P., J.-L.G.-S., and J.C.-M.; Project Administration, J.-L.G.-S. and J.C.-M. All authors have read and agreed to the published version of the manuscript.

Funding: This work was supported in part by the European Regional Development Fund, and in part by the Regional Ministry of Economy, Science and Digital Agenda of the Junta de Extremadura under Project GR18195. This research is also financed by the Regional Ministry of Economy and Infrastructure of the Junta de Extremadura under project IB18003 and the Spanish National Program of Research, Development and innovation under project RTI2018-102002-A-I00.

Acknowledgments: The authors are grateful to the Research, Technological Innovation and Supercomputing Center of Extremadura (CénitS) for allowing us to use their supercomputing facilities (LUSITANIA II).

Conflicts of Interest: The authors declare no conflicts of interest.

\section{References}

1. Cisco Systems. Cisco Visual Networking Index: Global Mobile Data Traffic Forecast Update, 2017-2022; White Paper; Cisco Public Information: San Jose, CA, USA, February 2019.

2. Nokia Siemens Networks. Signaling Is Growing 50\% Faster Than Data Traffic; White Paper; Nokia Siemens Networks: Espoo, Finland, 2012.

3. Perkins, C.; Johnson, D.; Arkko, J. Mobility Support in IPv6. RFC 6275, July 2011. Available online: https:/ / tools.ietf.org/html/rfc6275 (accessed on 25 February 2020).

4. Gundavelli, S.; Leung, K.; Devarapalli, V.; Chowdhury, K.; Patil, B. Proxy Mobile IPv6. RFC 5213, August 2008. Available online: http:/ /www.hjp.at/doc/rfc/rfc5213.html (accessed on 25 February 2020).

5. Carmona-Murillo, J.; Soto, I.; Rodríguez-Pérez, F.-J.; Cortés-Polo, D.; González-Sánchez, J.-L. Performance Evaluation of Distributed Mobility Management Protocols: Limitations and Solutions for Future Mobile Networks. Mob. Inf. Syst. 2017, 2017, 2568983. [CrossRef]

6. Carmona-Murillo, J.; Friderikos, V.; González-Sánchez, J.L. A hybrid DMM solution and trade-off analysis for future wireless networks. Comput. Netw. 2018, 133, 17-32. [CrossRef]

7. Jeon, S.; Figueiredo, S.; Aguiar, R.L.; Choo, H. Distributed Mobility Management for the Future Mobile Networks: A Comprehensive Analysis of Key Design Options. IEEE Access 2017, 5, 11423-11436. [CrossRef]

8. Ali-Ahmad, H.; Ouzzif, M.; Bertin, P.; Lagrange, X. Performance Analysis on Network-Based Distributed Mobility Management. Wirel. Pers. Commun. 2014, 74, 1245-1263. [CrossRef]

9. Kleinrock, L. Queueing Systems: Theory; Wiley: New York, NY, USA, 1975. 
10. Murtadha, M.K.; Noordin, N.K.; Ali, B.M.; Hashim, F. Design and evaluation of distributed and dynamic mobility management approach based on PMIPv6 and MIH protocols. Wirel. Netw. 2015, 21, 2747-2763. [CrossRef]

11. Makaya, C.; Pierre, S. An Analytical Framework for Performance Evaluation of IPv6-Based Mobility Management Protocols. IEEE Trans. Wirel. Commun. 2008, 7, 972-983. [CrossRef]

12. Akyildiz, I.; Wang, W. A dynamic location management scheme for next-generation multitier PCS Systems. IEEE Trans. Wirel. Commun. 2002, 1, 178-189. [CrossRef]

13. Wan, G.; Lin, E. Cost reduction in location management using semi-realtime movement information. ACM-Baltzer J. Wirel. Netw. 1999, 5, 245-256. [CrossRef]

14. Cortés-Polo, D.; González-Sánchez, J.L.; Carmona-Murillo, J.; Rodríguez-Pérez, F.-J. Proposal and analysis of integrated PTN architecture in the mobile backhaul to improve the QoS of HetNets. EURASIP J. Wirel. Commun. Netw. 2015, 2015, 116. [CrossRef]

15. Cortés-Polo, D.; Calle-Cancho, J.; Carmona-Murillo, J.; González-Sánchez, J. Future Trends in Mobile-Fixed Integration for Next Generation Networks: Classification and Analysis. Int. J. Veh. Telemat. Infotain. Syst. 2017, 1, 33-53. [CrossRef]

16. Lee, J.-H.; Bonnin, J.-M.; You, I.; Chung, T.-M. Comparative Handover Performance Analysis of IPv6 Mobility Management Protocols. IEEE Trans. Ind. Electron. 2013, 60, 1077-1088.

17. Bernardos, C.J.; De la Oliva, A.; Giust, F. A PMIPv6-Based Solution for Distributed Mobility Management; draft-bernardos-dmm-pmip-09; IMDEA Networks Institute: Madrid, Spain, 2017.

18. CORE. Common Open Research Emulator. Available online: https://www.nrl.navy.mil/itd/ncs/products/ core (accessed on 6 February 2020).

(C) 2020 by the authors. Licensee MDPI, Basel, Switzerland. This article is an open access article distributed under the terms and conditions of the Creative Commons Attribution (CC BY) license (http:/ / creativecommons.org/licenses/by/4.0/). 